\title{
New Type of Difference Sequence Spaces of Fuzzy Real Numbers
}

\author{
B. C. Tripathy ${ }^{1}$ and A. Baruah ${ }^{2}$ \\ ${ }^{1}$ Mathematical Sciences Division; Institute of Advanced Study in Science \\ and Technology; Paschim Boragaon \\ ${ }^{2}$ Department of Mathematics; North Gauhati College; College Nagar \\ ${ }^{1}$ Garchuck; Guwahati-781035; India \\ ${ }^{2}$ Guwahati-781031; India \\ E-mail(corresp.): achuta_math@rediffmail.com \\ E-mail: tripathybc@yahoo.com;tripathybc@rediffmail.com
}

Received March 20, 2007; revised November 6, 2007; published online July 15, 2009

\begin{abstract}
In this paper we introduce the natation difference operator $\Delta_{m}(m \geq$ 0 , an integer) for studying properties of some sequence spaces. We define the sequence spaces $\ell_{\infty}^{F}\left(\Delta_{m}\right), c^{F}\left(\Delta_{m}\right), c_{0}^{F}\left(\Delta_{m}\right)$ and investigate their properties like solidness, convergence free, symmetricity, completeness.
\end{abstract}

Key words: Fuzzy sequences, difference sequence space, solid space, symmetric space, convergence free, completeness.

\section{Introduction}

The concept of fuzzy set was introduced by Zadeh [11]. Bounded and convergent sequences of fuzzy numbers were studied by Matloka [6], where it was shown that every convergent sequence is bounded. Later, different classes of sequences of fuzzy numbers have been studied by Esi [2], Tripathy and Nanda [10], Savas [7], Fang and Hung [3], Choudhary and Tripathy [1], Tripathy and Borgohain [8].

Let $D$ denote the set of all closed and bounded intervals $X=\left[a_{1}, a_{2}\right]$ on the real line $R$. For $X, Y \in D$ we define

$$
d(X, Y)=\max \left(\left|a_{1}-b_{1}\right|,\left|a_{2}-b_{2}\right|\right) \text { where } X=\left[a_{1}, a_{2}\right], Y=\left[b_{1}, b_{2}\right] .
$$

It is known that $(D, d)$ is a complete metric space.

Let $I=[0,1]$. A fuzzy real number $X$ is a fuzzy set on $R$ and is a mapping $X: R \rightarrow I$ associating each real number $t$ with its grade member ship $X(t)$.

A fuzzy real number $X$ is called convex if

$$
X(t) \geq X(s) \wedge X(r)=\min (X(s), X(r)), \text { where } s<t<r .
$$


A fuzzy real number $X$ is called normal if there exists $t_{0} \in R$, such that $X\left(t_{0}\right)=1$. A fuzzy real number $X$ is said to be upper semi-continuous if for each $\varepsilon>0, X^{-1}([0, a+\varepsilon))$, for all $a \in I$ and given $\varepsilon 0, X^{-1}([0, a+\varepsilon))$, is open in the usual topology of $R$.

The set of all upper-semi continuous, normal, convex fuzzy numbers is denoted by $R(I)$. The $\alpha$-level set of a fuzzy real number $X$, for $0<\alpha \leq 1$ denoted by $X^{\alpha}$ is defined as $X^{\alpha}=\{t \in R: X(t) \geq \alpha\}$. The 0-level set is the closure of strong 0 -cut.

For each $r \in R, \bar{r} \in R(I)$ is defined by

$$
\bar{r}(t)= \begin{cases}1, & \text { if } t=r \\ 0, & \text { if } t \neq r .\end{cases}
$$

The absolute value $|X|$ of $X \in R(I)$ is defined by (see for instance Kaleva and Seikkla [4])

$$
|X|(t)= \begin{cases}\max \{X(t), X(-t)\}, & \text { for } t \geq 0 \\ 0, & \text { for } t<0 .\end{cases}
$$

Let $\bar{d}: R(I) \times R(I) \rightarrow R$ be defined by

$$
\bar{d}(X, Y)=\sup _{0 \leq \alpha \leq 1} d\left(X^{\alpha}, Y^{\alpha}\right) .
$$

Then $\bar{d}$ defines a metric on $R(I)$ (Matloka [6]). The additive identity and multiplicative identity in $R(I)$ are denoted by $\overline{0}$ and $\overline{1}$ respectively.

In this paper we have introduced the classes of the difference sequences $c^{F}\left(\Delta_{m}\right), c_{0}^{F}\left(\Delta_{m}\right)$ and $\ell_{\infty}^{F}\left(\Delta_{m}\right)$ of fuzzy real numbers. We have shown that these are complete metric spaces. We have investigated different properties like solidness, symmetricity and convergence free of these spaces.

\section{Definitions and Preliminaries}

Throughout the article $w^{F}, c^{F}, c_{0}^{F}, \ell_{\infty}^{F}$ denote the classes of all, convergent, null, bounded sequence spaces of fuzzy real numbers.

A fuzzy real valued sequence $\left\{X_{n}\right\}$ is said to be convergent to the fuzzy real number $X$, if for $\varepsilon>0$, there exists $n_{o} \in N$ such that $\bar{d}\left(X_{k}, X\right)<\varepsilon$, for all $k \geq n_{0}$ (Matloka [6]).

A sequence space $E^{F}$ is said to be solid (or normal) if $\left(X_{k}\right) \in E^{F}$ implies that $\left(\alpha_{k} X_{k}\right) \in E^{F}$ for all sequences of scalars $\left(\alpha_{k}\right)$ with $\left|a_{k}\right| \leq 1$, for all $k \in N$.

Let $K=\left\{k_{1}<k_{2}<\ldots\right\} \subseteq N$ and $E^{F}$ be a sequence space. A $K$-step space of $E^{F}$ is a sequence space $\lambda_{K}^{E^{F}}=\left\{\left(X_{k_{n}}\right) \in w^{F}:\left(X_{n}\right) \in E^{F}\right\}$.

A canonical preimage of a sequence $\left\{X_{k}\right\} \in \lambda_{K}^{E^{F}}$ is a sequence $\left\{Y_{n}\right\} \in w^{F}$ defined as

$$
Y_{n}= \begin{cases}X_{n}, & \text { if } n \in K \\ \overline{0}, & \text { otherwise. }\end{cases}
$$

A canonical preimage of a step space $\lambda_{K}^{E^{F}}$ is a set of canonical preimages of all elements in $\lambda_{K}^{E^{F}}$, i.e. $Y$ is in canonical preimage of $\lambda_{K}^{E^{F}}$ if and only if $Y$ is canonical preimage of some $X \in \lambda_{K}^{E^{F}}$. 
A sequence space $E^{F}$ is said to be monotone if it contains the canonical preimages of its step spaces.

A sequence space $E^{F}$ is said to be convergence free if $\left(Y_{k}\right) \in E^{F}$ whenever $\left(X_{k}\right) \in E^{F}$ and $Y_{k}=\overline{0}$ whenever $X_{k}=\overline{0}$.

Kizmaz [5] defined the difference sequence space for crisp set. This concept was further generalized by Tripathy and Esi [9] as follows. Let $m \geq 0$ be an integer then $Z\left(\Delta_{m}\right)=\left\{\left(x_{k}\right) \in w:\left(\Delta_{m} x_{k}\right) \in Z\right\}$, for $Z=c, c_{0}, \ell_{\infty}$ where $\Delta_{m} x_{k}=x_{k}-x_{k+m}$, for all $k \in N$. For $m=1$, the spaces $\ell_{\infty}(\Delta), c(\Delta), c_{0}(\Delta)$ are studied by Kizmaz [5]. The idea of Kizmaz [5] was applied by Savas [7] for introducing the notion of difference sequences for fuzzy real numbers and study their different properties.

We introduce the following difference sequences of fuzzy real numbers of Tripathy \& Esi [9] type as follows. Let $m \geq 0$ be an integer then,

$$
Z\left(\Delta_{m}\right)=\left\{\left(X_{k}\right) \in w^{F}:\left(\Delta_{m} X_{k}\right) \in Z\right\}, \quad \text { for } Z=c^{F}, c_{0}^{F}, \ell_{\infty}^{F},
$$

where $\Delta_{m} X_{k}=X_{k}-X_{k+m}$, for all $k \in N$.

\section{Main Results}

Theorem 1. The sequence spaces $c^{F}\left(\Delta_{m}\right), c_{0}^{F}\left(\Delta_{m}\right)$ and $\ell_{\infty}^{F}\left(\Delta_{m}\right)$ are complete metric spaces by the metric

$$
\rho(X, Y)=\sum_{k=1}^{m} \bar{d}\left(X_{k}, Y_{k}\right)+\sup _{k} \bar{d}\left(\Delta_{m} X_{k}, \Delta_{m} Y_{k}\right) .
$$

Proof. Let $\left(X^{i}\right)$ be any Cauchy sequence in $\ell_{\infty}^{F}\left(\Delta_{m}\right)$ where $X^{i}=\left(X_{k}^{i}\right)=$ $\left(X_{1}^{i}, X_{2}^{i}, X_{3}^{i}, \ldots\right) \in \ell_{\infty}^{F}\left(\Delta_{m}\right)$ for each $i \in N$. Then for given $\varepsilon \geq 0$, there exist $n_{0} \in N$ such that

$$
\rho\left(X^{i}, Y^{j}\right)=\sum_{k=1}^{m} \bar{d}\left(X_{k}^{i}, Y_{k}^{j}\right)+\sup _{k} \bar{d}\left(\Delta_{m} X_{k}^{i}, \Delta_{m} Y_{k}^{j}\right)<\varepsilon, \text { for } i, j \geq n_{0} .
$$

Hence

$$
\begin{aligned}
\sum_{k=1}^{m} \bar{d} & \left(X_{k}^{i}, X_{k}^{j}\right)<\varepsilon, \text { for all } i, j \geq n_{0} \text { and } k=1,2,3, \ldots m \\
& \Longrightarrow \bar{d}\left(X_{k}^{i}, Y_{k}^{j}\right)<\varepsilon, \text { for all } i, j \geq n_{0} \text { and } k=1,2,3, \ldots m \\
& \Longrightarrow\left(X_{k}^{j}\right) \text { is a Cauchy sequence in } R(I) \text { for } k=1,2,3, \ldots m \\
& \Longrightarrow\left(X_{k}^{j}\right) \text { is a convergent sequence in } R(I) \text { for } k=1,2,3, \ldots m
\end{aligned}
$$

Let $\lim _{j \rightarrow \infty} X_{k}^{j}=X_{k}$ (say) for $k=1,2,3, \ldots m$. Again from $(3.1)$

$$
\bar{d}\left(\Delta_{m} X_{k}^{i}, \Delta_{m} X_{k}^{j}\right)<\varepsilon, \text { for } i, j \geq n_{0} \text { for } k \in N
$$

$\Longrightarrow\left(\Delta_{m} X_{k}^{j}\right)$ is a Cauchy sequence in $R(I)$ for all $k \in N$

$\Longrightarrow\left(\Delta_{m} X_{k}^{j}\right)$ is a convergent sequence in $R(I)$ for all $k \in N$. 
Let $\lim _{j \rightarrow \infty} \Delta_{m} X_{k}^{j}=Y_{k}$ for each $k \in N$. Since $\lim _{j \rightarrow \infty} X_{k}^{j}=X_{k}$, for $k=1, \ldots m$, then $\lim _{j \rightarrow \infty} X_{k}^{j}=X_{k}$ exist for each $k \in N$. For all $i \geq n_{0}$, we have

$$
\begin{aligned}
& \lim _{j \rightarrow \infty} \sum_{k=1}^{m} \bar{d}\left(X_{k}^{i}, X_{k}^{j}\right)=\sum_{k=1}^{m} \bar{d}\left(X_{k}^{i}, X_{k}\right)<\varepsilon \\
& \lim _{j \rightarrow \infty} \bar{d}\left(\Delta_{m}\left(X_{k}^{i}, \Delta_{m} X_{k}^{j}\right)=\bar{d}\left(\Delta_{m} X_{k}^{i}, \Delta_{m} X_{k}\right)<\varepsilon .\right.
\end{aligned}
$$

Hence for all $i \geq n_{0}$, it follows that

$$
\sup _{k} \bar{d}\left(\Delta_{m} X_{k}^{i}, \Delta_{m} X_{k}\right)<\varepsilon .
$$

Thus for all $i, j \geq n_{0}$ we obtain

$$
\sum_{k=1}^{m} \bar{d}\left(X_{k}^{i}, X_{k}\right)+\sup _{k} \bar{d}\left(\Delta_{m} X_{k}^{i}, \Delta_{m} X_{k}\right)<2 \varepsilon \quad \Longrightarrow \quad \rho\left(X^{i}, X\right)<2 \varepsilon
$$

i.e. $X^{i} \rightarrow X$, as $i \rightarrow \infty$ in $\ell_{\infty}^{F}\left(\Delta_{m}\right)$ follows. For $i \geq n_{0}$, we have

$$
\sup _{k} \bar{d}\left(\Delta_{m} X_{k}, \overline{0}\right) \leq \sup _{k} \bar{d}\left(\Delta_{m} X_{k}, \Delta_{m} X_{k}^{i}\right)+\sup _{k} \bar{d}\left(\Delta_{m} X_{k}^{i}, \overline{0}\right)<\infty .
$$

This completes the proof.

Result 2. The spaces $c^{F}\left(\Delta_{m}\right), c_{0}^{F}\left(\Delta_{m}\right)$ and $\ell_{\infty}^{F}\left(\Delta_{m}\right)$ are not solid in general.

Proof. The result follows from the following example:

Example 1. Consider the sequence $\left(X_{n}\right)$ defined by

$$
X_{n}(t)= \begin{cases}\frac{n t+n+1}{n+1}, & \text { for }-1-\frac{1}{n} \leq t \leq 0 \\ \frac{n+1-n t}{n+1}, & \text { for } 0 \leq t \leq 1+\frac{1}{n} \\ 0, & \text { otherwise. }\end{cases}
$$

For $m=3$ we have that

$$
\Delta_{3} X_{n}(t)= \begin{cases}\frac{t n^{2}+2 n^{2}+3 n t+8 n+3}{2 n^{2}+8 n+3}, & \text { for }-2-\frac{1}{n}-\frac{1}{n+3} \leq t \leq 0, \\ \frac{-t n^{2}-3 t n+2 n^{2}+8 n+3}{2 n^{2}+8 n+3}, & \text { for } 0 \leq t \leq 2+\frac{1}{n}+\frac{1}{n+3} \\ 0, & \text { otherwise. }\end{cases}
$$

Now $\lim _{n \rightarrow \infty} \Delta_{3} X_{n}=X$, where

$$
X(t)= \begin{cases}\frac{t+2}{2}, & \text { for }-2 \leq t \leq 0 \\ \frac{2-t}{2}, & \text { for } 0 \leq t \leq 2 \\ 0, & \text { otherwise. }\end{cases}
$$


Thus $\left(X_{n}\right) \in c^{F}\left(\Delta_{3}\right)$. Now consider the sequence of scalars $\left(\alpha_{n}\right)$ defined by

$$
\left(\alpha_{n}\right)= \begin{cases}1, & \text { for } n=3 k-2, \text { for } k \in N \\ 0, & \text { otherwise }\end{cases}
$$

Then $\left(\alpha_{n} X_{n}\right)=\left\{X_{1}, \overline{0}, \overline{0}, X_{4}, \overline{0}, \overline{0}, X_{7}, \overline{0}, \overline{0}, X_{10}, \ldots\right\}$. But

$$
\left(\Delta_{3} \alpha_{n} X_{n}\right)=\left\{X_{1}-X_{4}, \overline{0}, \overline{0}, X_{4}-X_{7}, \overline{0}, \overline{0}, \ldots\right\} \notin c^{F}\left(\Delta_{3}\right) .
$$

Hence $c^{F}\left(\Delta_{m}\right)$ is not solid.

Similar examples can be constructed for the other spaces.

Result 3. The space $c^{F}\left(\Delta_{m}\right), c_{0}^{F}\left(\Delta_{m}\right)$ and $\ell_{\infty}^{F}\left(\Delta_{m}\right)$ are not symmetric in general.

Proof. The result follows from the following example.

Example 2. Let $m=1$, and consider the sequence $X=\{A, B, A, B, A, B, \ldots\}$, where

$$
A=\left\{\begin{array}{ll}
\frac{t+4}{4}, & \text { for }-4 \leq t \leq 0, \\
\frac{4-t}{4}, & \text { for } 0 \leq t \leq 4, \\
0, & \text { otherwise. }
\end{array} \quad, \quad B= \begin{cases}\frac{t+5}{5}, & \text { for }-5 \leq t \leq 0 \\
\frac{5-t}{5}, & \text { for } 0 \leq t \leq 5 \\
0, & \text { otherwise }\end{cases}\right.
$$

Now consider the re-arrangement $\left(Y_{n}\right)$ of the sequence $\left(X_{n}\right)$ as

$$
\left(Y_{n}\right)=\{A, A, B, B, A, A, \ldots\} \notin c^{F}(\Delta), \text { but }\left(X_{n}\right) \in c^{F}(\Delta) .
$$

Hence $c^{F}\left(\Delta_{m}\right)$ is not Symmetric for any $m \in N$.

Similar examples can be constructed for the other spaces.

Result 4. The spaces $c^{F}\left(\Delta_{m}\right), c_{0}^{F}\left(\Delta_{m}\right)$ and $\ell_{\infty}^{F}\left(\Delta_{m}\right)$ is not convergence free.

Proof. The result follows from the following example.

Example 3. Consider the sequence $\left(X_{n}\right)$ defined as follows

$$
X_{n}(t)= \begin{cases}\frac{n t+n+1}{n+1}, & \text { for }-1-\frac{1}{n} \leq t \leq 0 \\ \frac{n+1-n t}{n+1}, & \text { for } 0 \leq t \leq 1+\frac{1}{n} \\ 0, & \text { otherwise }\end{cases}
$$


Now we obtain

$$
\Delta_{3} X_{n}(t)= \begin{cases}\frac{t n^{2}+2 n^{2}+3 n t+8 n+3}{2 n^{2}+8 n+3}, & \text { for }-2-\frac{1}{n}-\frac{1}{n+3} \leq t \leq 0, \\ \frac{-t n^{2}-3 n t+2 n^{2}+8 n+3}{2 n^{2}+8 n+3}, & \text { for } 0 \leq t \leq 2+\frac{1}{n}+\frac{1}{n+3}, \\ 0, & \text { otherwise }\end{cases}
$$

and $\lim _{n \rightarrow \infty} \Delta_{3} X_{n}=X$ defined as follows

$$
X(t)= \begin{cases}\frac{t+2}{2}, & \text { for }-2 \leq t \leq 0 \\ \frac{2-t}{2}, & \text { for } 0 \leq t \leq 2 \\ 0, & \text { otherwise }\end{cases}
$$

Thus $\left(X_{n}\right) \in c^{F}\left(\Delta_{3}\right)$. Now consider

$$
Y_{n}(t)= \begin{cases}\frac{t+n}{n}, & \text { for }-n \leq t \leq 0 \\ \frac{n-t}{n}, & \text { for } 0 \leq t \leq n \\ 0, & \text { otherwise }\end{cases}
$$

where

$$
\Delta_{3} Y_{n}(t)= \begin{cases}\frac{t+2 n+3}{2 n+3}, & \text { for }-2 n-3 \leq t \leq 0, \\ \frac{2 n+3-t}{2 n+3}, & \text { for } 0 \leq t \leq 2 n+3, \\ 0, & \text { otherwise. }\end{cases}
$$

Clearly $\left(Y_{n}\right) \in c^{F}\left(\Delta_{3}\right)$. Therefore the space $c^{F}\left(\Delta_{3}\right)$ is not convergence free.

Similar examples can be constructed for other spaces.

\section{Conclusions}

Following the notion of difference operator $\Delta_{m}$, introduced by Tripathy and Esi [7], the difference sequences $c^{F}\left(\Delta_{m}\right), c_{0}^{F}\left(\Delta_{m}\right)$ and $\ell_{\infty}^{F}\left(\Delta_{m}\right)$ of fuzzy numbers have been introduced. It is shown that these classes of sequences are complete metric spaces. It is observed that these classes are neither solid nor convergence free nor symmetric. The introduced notion of difference operator can be applied for studying many other classes of sequences of fuzzy numbers.

\section{Acknowledgement}

The authors thank the referee for his careful reading of the manuscript and the suggestions. 


\section{References}

[1] B. Choudhary and B.C. Tripathy. On fuzzy real-valued $\ell(p)^{F}$ sequences. In Proc. International Conf. 8th Joint Conf. Inf. Sci. (10th International Conf. on Fuzzy Theorey and Technology, pp. 184-190, Saltlake City, Utah, USA, July 21-25, 2005.

[2] A. Esi. On some new paranormed sequence spaces of fuzzy numbers defined by Orlicz functions and statistical convergence. Math. Model. Anal., 11(4):379-388, 2006 .

[3] J.X. Fang and H. Hung. On the level convergence of a sequence of fuzzy numbers. Fuzzy Sets and Systems, 147:417-435, 2004.

[4] O. Kelava and S. Seikkala. On fuzzy metric spaces. Fuzzy Sets and Systems, 12:215-229, 1984.

[5] H. Kizmaz. On certain sequence spaces. Canad. Math. Bull., 24(2):168-176, 1981.

[6] M. Matloka. Sequences of fuzzy numbers. BUSEFAL, 28:28-37, 1986.

[7] E. Savas. A note on sequence of fuzzy numbers. Information Sciences, 124:297$300,2000$.

[8] B.C. Tripathy and S. Borgohain. The sequence space $m\left(M, \phi, \Delta_{m}^{n}, p\right)^{F}$. Math. Model. Anal., 13(4):577-586, 2008. (doi:10.3846/1392-6292.2008.13.577-586 )

[9] B.C. Tripathy and A. Esi. A new type of difference sequence spaces. International Journal of Sci. Tech., 1(1):11-14, 2006.

[10] B.K. Tripathy and S. Nanda. Absolute value of fuzzy real numbers and fuzzy sequence spaces. J. Fuzzy Math., 8(4):883-892, 2000.

[11] L.A. Zadeh. Fuzzy sets. Information and Control, 8(3):338-353, 1965. 